\title{
Quantitative Determination of Nicotine in a PDMS Microfluidic Channel Using Surface Enhanced Raman Spectroscopy
}

\author{
Jaehyun Jung, Jaebum Choo, ${ }^{\star}$ Duck Joong Kim, ${ }^{\dagger}$ and Sanghoon Lee ${ }^{\ddagger}$ \\ Department of Applied Chemistry. Hanvang Lniversity, Ansan 426-791, Korea. "E-mail: jbchoo@ hanang.ackr. \\ "Department of Biomedical Engineering. Dankook Universin; Cheonan 330-714. Korea \\ 'Department of Biomedical Engineering, Korea University, Seonl 136-701, Korea \\ Received December 6, 2005
}

\begin{abstract}
Rapid and highly sensitive determination of nicotine in a PDMS microfluidic channel was investigated using surface enhanced Raman spectroscopy (SERS). $\Lambda$ three-dimensional PDMS mictofluidic channel was fabricated for this purpose. This channel shows a high mixing efficiency because the transverse and vertical dispersions of the fluid occur sumultaneously through the upper and lower zig zag-type blocks. $\Lambda$ higher effíciency of mixing could also be obtained by splitting each of the confluent streams into two sub-streanns that then joined and recombined. The SERS signal was measured atter nicotine molecules were effectively adsorbed onto silver nanoparticles by passing through the three-dimensional channel. $\Lambda$ quantitative analysis of nicotine was performed based on the measured peak area at $1030 \mathrm{~cm}^{-1}$. The detection limit was estimated to be below $0.1 \mathrm{ppm}$. In this work, the SERS detection, in combination with a PDMS microfluidic channel, has been applied to the quantitative analysis of nicotine in aqueous solution. Compared to the other conventional analytical methods, the detection sensitivity was enhanced up to several orders of magnitude.
\end{abstract}

Key Words : Surface enhanced Raman spectroscopy, Microfluidic chip, Nicotine, Quantitative analysis

\section{Introduction}

Nicotine is one of the most potent pharmacological agents in tobacco and tobacco smoke, hence, it is of considerable interest to medicine and society. ${ }^{1}$ A quantitative analysis of nicotine has been used to study the smoking behavior of smokers and the quality control of nicotine-containing products as well as the determination of nicotine amount in biological fluids. ${ }^{2.3}$ various analytical methods, such as HPLC, ${ }^{4}$ UV/Vis spectroscopy ${ }^{5}$ GC/MS, ${ }^{6}$ radioimmunoassay, ${ }^{7}$ chemosensor ${ }^{8}$ and antibody/antigen sensor, ${ }^{9}$ have been applied to the quantitative analysis of nicotine. For example, GC/MS has been used to quantify the nicotine present in the hair of smokers and non-smokers. However, some of the problems associated with the previously reported analytical methods, such as a long sample preparation time, poor detection limit, lengthy measurement time and indirect measurement, made the detection systems less attractive.

Surface enlhanced Raman spectroscopy (SERS) has also been used for the quantitative analysis of nicotine. Previous SERS studies of nicotine have used silver-coated alumina substrates, ${ }^{10}$ silver nano particle doped cellulose acetate membranes, ${ }^{11}$ and electrochemically roughened electrodes. ${ }^{12}$ More recently, Bell and Sirimuthul $1^{13}$ reported sensitive nicotine detection results using polymer-encapsulated silver nanoparticles. These nanoparticles were used for quantitative determination of unknown nicotine samples at the 1-5 ppm level. However, the quantitative application of SERS is known to be very challenging because it is difficult to control the experimental conditions such as the degree of aggregation, the particle sizes of the metal colloids, and the inhomogeneous distribution of molecules on the metal surface ${ }^{1+.15}$ As a result, under ordinary sampling conditions, the expected precision from SERS is very poor. Nonetheless, a highly precise quantitative measurement can be obtained if continuous flow and homogeneous mixing conditions between the analytes and a silver nanocolloid are maintained. For this purpose, we used a polydimethylsiloxane (PDMS) microfluidic device, combined with a highly sensitive SERS detection. Recently, we have successfully used this SERS technique for the rapid and highly sensitive detection of duplex dye labeled DNA sequences, ${ }^{16}$ cyanide ion water pollutants, ${ }^{17}$ and methyl parathion pesticides in a PDMS microfluidic channel..$^{18}$ In the present study, we extend its applicability to the trace analysis of nicotine.

\section{Experimental Section}

Silver Colloid Preparation. Silver colloids were prepared by the method recently reported by Leopold and Lendl. ${ }^{19}$ Here, silver nitrate was reduced by hydroxylamine hydrochloride. The advantages of the hydroxylamine hydrochloride-reduced silver colloid are its fast preparation at room temperature and its immediate applicability for SERS. The detailed procedure for the preparation of silver nanoparticles has been reported elsewhere. ${ }^{16}$ UV/Vis spectroscopy and transmission electron microscopy (TEM) were used to characterize the particle size of the produced colloids. The average particle size was determined to be 60 nm.

Fabrication of the PDMS Microfluidic Channel. The fabrication process of zig zag-type PDMS microfluidic channel has been reported elsewhere. ${ }^{20.21}$ Briefly, the microfluidic channel were fabricated stacking two PDMS layers 
having upper and lower rig zag-type block patterns. These layers were produced by pattern replication from mold masters. The cover glass was stacked onto the upper layer. Within a rectangular duet, the bar struetures were located on upper and lower surfaees of the channel in a rig zag manner.

Surface Fnhaned Raman Measurements. Raman measurements were performed with a Renishaw 2000 Raman mieroscope system. A Spectra Physics argon ion laser operating at $\lambda=514.5 \mathrm{~mm}$ was used as the excitation source with a laser power of approximately $20 \mathrm{~mW}$. The Rayleigh line was removed from the collected Raman seattering by a holographic notch filter in the collection path. A charge coupled device (CC.D) camera was coupled to a spectrograph that in combination provided a $2 \mathrm{~cm}^{-1}$ spectral resolution. All spectral manipulations were performed using $G R A M / 32$ software from Galactic Industries Corporation, C.S.A.

Confocal Fluorescence Measurements. Confocal fluorescence intage measurements were performed using a I.eica TCS SP confocal fluoreseence microseope with a $\mathrm{He}-\mathrm{Ne}$ laser. Fluoreseent Rhodamine 6G dissolved in water and non-fluoreseent silver colloids were introdueed through a $\mathrm{Y}$ shaped inlel using mierosyringe pumps to display the mixing process of the confluent streans. The confluent mixing streams were analyzed using the two-dimensional confocal fluoresenes images in the $x-y$ plane located perpendicular to the optical axis using a $10 \times$ water-immersion objective lens. The lateral resolution was estimated to be $1 \mathrm{~mm}$. The laser excitation of Rhodamine $6 \mathrm{G}$ occurred at $\lambda=543 \mathrm{~nm}$, and the emitted fluorescent light was detected between $\lambda=$ 590 and $620 \mathrm{~nm}$. The image size was $512 \times 512$ pixels, and each pixel was $0.49 \mu \mathrm{m}$. The fluoreseence intensity profiles of the llow across the channel were also measured to monitor the mixing behavior of the confluent mixing streams.

\section{Results and Discussion}

Figure 1 shows a schematic drawing of the PDMS micromixer and the mixing process between the silver nanocolloid and the nicotine in the microfluidic channel. The silver colloids and the different concentrations of nicotine were introduced into the channel using microsyringes connected by tubes to the inlet pipettes. The flow rate was simultaneously controlled using a microsyringe pump. While the confluent streams travel along the microfluidic channel, the transverse and vertical dispersions of the fluid occur simultaneously through the upper and lower zig zag-type blocks. The confluent stream was split into two lines to improve the mixing efficiency. A high efficiency of mixing could be obtained by splitting each of the confluent streams into two sub-streams that then joined and recombined. Heule and Manz $z^{22}$ previously reported that the problems of diffusion limitations in the laminar flow regime can be overcome using the multi-splitting channel. In our work, the double split design as well as the zig zag-type chaotic advection design was adopted for obtaining high mixing efficiency. After the nicotine molecules were effectively adsorbed onto

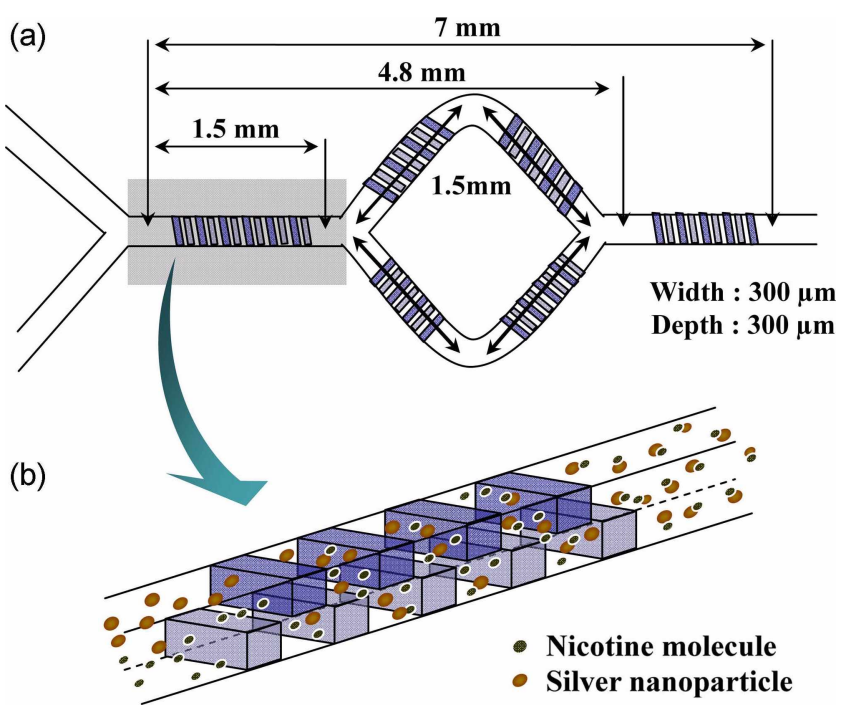

Figure 1. (a) A schematic view of the zig zag-type PDMS microfluidic channel. (b) A mixing process of confluent streams, colloidal silver nanoparticles, and nicoline solution. While laminar flow streams travesse the microfluidie channel, the transverse and vertical dispersion of the fluid occurs simultaneously through the upper and lower blocks.

the silver nanoparticles on traveling along the blocks and split channel lines, the STRS signal was measured.

In order to investigate the mixing efliciency of the channel used, the fluoresecnec image was measured using a confocal laser scanning microscope. Iaser-grade Rhodamine $6 \mathrm{G}$ was dissolved in distilled water to produce a $0.05 \mathrm{M}$ solution that formed one of the confluent streams in the channel. The other stream contained silver colloids. These two streans were introduced into the PDMS channel from microsyringes connected by the tubes to the inlet pipettes. The flow rates were controlled simultaneously using a KD Science microsyringe pump. Figure 2 shows the captured lluoreseence inages and corresponding intensity profiles at the location
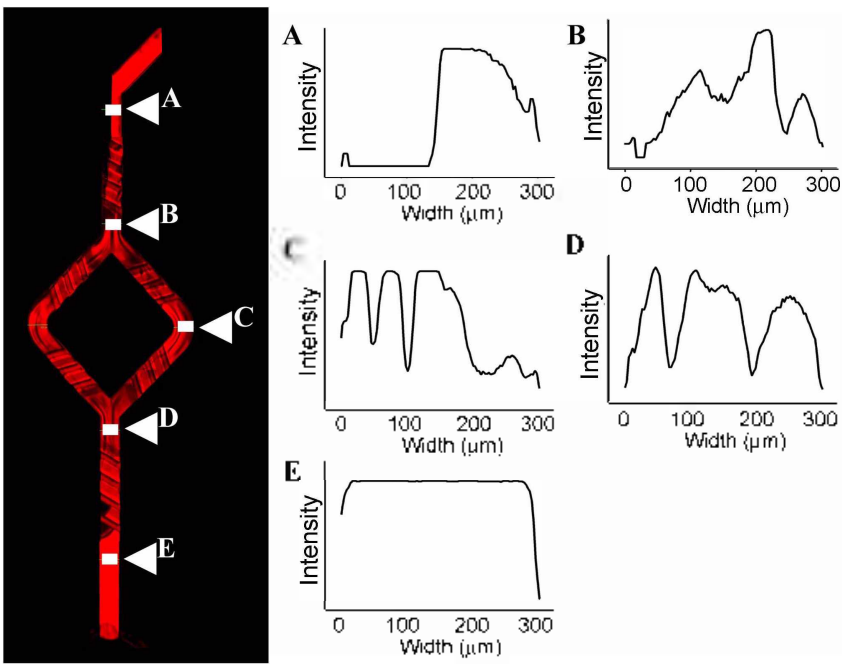

Figure 2. Confocal fluorescence images of lateral confluent streams along the channel and corresponding intensity protiles at the locations indicated by the solid lines. 


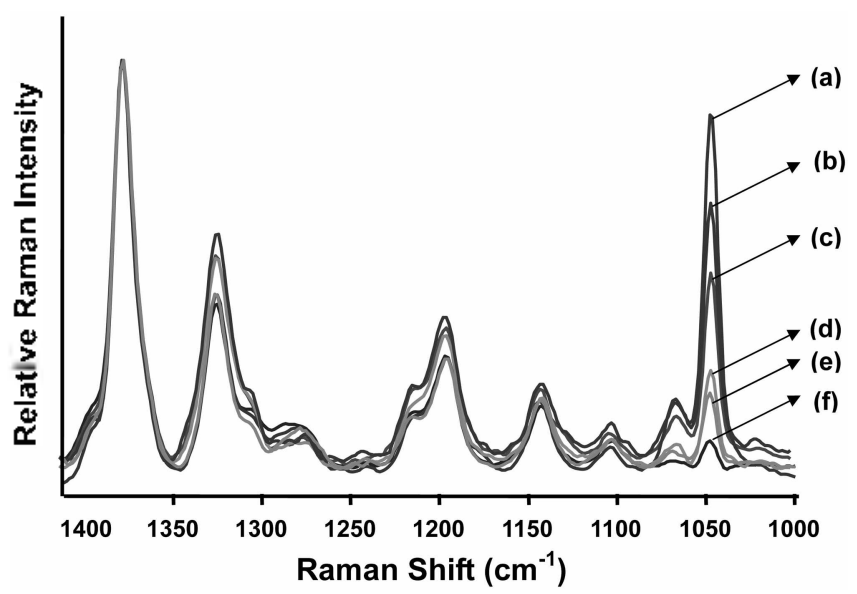

Figure 3. Confocal Sl:R spectra for varing concentralions of nicotine in the microfluidic channel: (a) $10 \mathrm{ppm}$. (b) $7.5 \mathrm{ppm}$, (c) $5.0 \mathrm{ppm}$, (d) $2.5 \mathrm{ppm}$, (e) $1.0 \mathrm{ppm}$, and (f) $0.1 \mathrm{ppm}$.

indicated by dashed lines at five different channel positions. The confluent streans display a heterogencous mixing behavior with increasing channel distance by the chaotic advection. To detemine the optimum conditions for the SFRS measurements, the flow rate of two confluent streams was varied in the range $1-170 \mu \mathrm{L} / \mathrm{min}$. According to our experimental data, the optimum rate was determined to be $100 \mu \mathrm{L} / \mathrm{min}$. As shown in Figure 2, the mixing of the red fluoreseence dye occurred from half of the chamel (A), to over the full channel ( $\Gamma$ ) along the channel distance. This means that the laninar flow mixing was efficiently driven by the chaotic advection as well as the splitting into two substreams. As a result, the confluent streams were completely mixed at the end of the channel ( $\Gamma$ ).

Figure 3 illustrates the STR spectra for different coneentrations of nicotine solution in a PDMS microfluidic chamel. The characteristic Raman peak of nicotine at 1030 $\mathrm{cm}^{-1}$ was monitored at different concentrations. The areas of the Raman peaks increased concomitantly with the increase in the concentration of nicotine. This peak was used for the

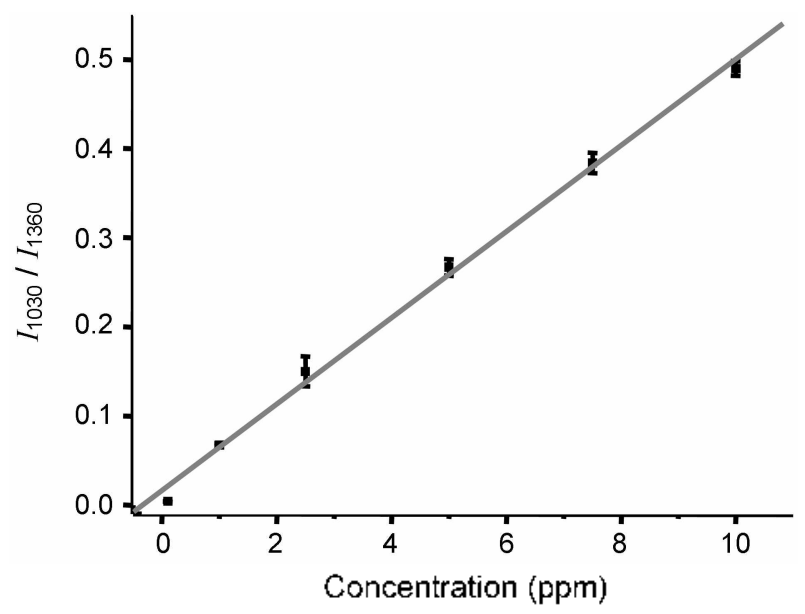

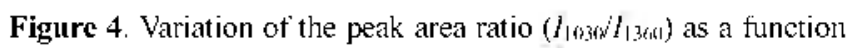
of nicotine concentration. (Correlation coefficient, $R=0.998$ ) quantitative determination of nicotine. In order to correct the sample-to-sample tluctuations in the Raman intensities, ${ }^{\text {?3.?.1 }}$ the Raman peak at $1030 \mathrm{~cm}^{-1}$ was normalized to the peak at $1360 \mathrm{~cm}^{-1}$. This is the peak for the reducing agent, hydroxylamine, and is used as an internal standard.

The calibration curve is shown in Figure 4. A very good linear response of peak area ratio $\left(\mathrm{I}_{1030 / 3} / \mathrm{I}_{1360)}\right)$ was found in the concentration range $0.1-10 \mathrm{ppm}$. The limit of detection (I.OJ) was determined to be below 0.1 ppm assessed from six standard deviations above the background. This means that the highly sensitive quantitative determination of nicoline in a PDMS microfluidic channel is possible using the surlace enhaneed Raman technicue.

\section{Conclusions}

Jab-on-a-chip technology is attracting great interest as the miniaturization of reaction systems offers practical advantages. Our proposed analytical method, using a PDMS microlluidic channel and a SГRS detection technicue, offers rapid and highly sensitive detcetion applicable to the quantiative determination of nicotine. Rapid mixing of the fluids flowing through a microchannel is very important for various applications of microfluidic systems. In addition, highly sensitive on-chip detection techniques are essential for the in situ monitoring of chemical reactions because the detection volume in a channel is extremely small. For this purpose, a rig rag-shaped split PDMS channel was fabricated. In this channel, the problems of diffusion limitations in the laminar flow could be overeome using the double split and rig rag- lype chaolic advection design. According to our fluoresence iminges, measured using a confocal laser scanning microscope, the confluent streams display a complete mixing behavior after they pass through the rig rag-type PDMS blocks. The STRS detection echnique has also been used for the highly sensitive analyte detection. In this work, the SERS detection, in combination with a PDMS microfluidic channel, has been applied to the quantitative analysis of nicotine in its aqueous solution. Compared to the other conventional analytical methods, the detection sensitivity was enhanced up to several orders of magnitude.

Acknowledgement. This work was supported by the Korea Research Foundation (Grant number R14-2002-00401000). J.C. also thanks Hanyang University for financial support during the 2004 academic year.

\section{References}

I. Banon, A. W; Decker. M. W; Holladay, M. W; Curzon, P.; Donnelly-Roberts, D.; Puttfarcken. P. S.: Bitner, R. S.; Diaz, A.: Dickenson, A. Il.; Porsolt, R. D.: Williams, M.; Arneric, S. P. Science 1998, 279, 77.

2. Iolladay, M. W.; Dart. M. J.; Lynch, J. K. .. Med. Chem. 1997 , 40, 4169 .

3. Elgen, R. M.; Hunter, J. C.: Dray, A. Thendis Phumacol. Sci. $1999,20,337$. 
4. Watson, I. D. J. Chromatogr. 1977, 143, 203.

5. Schmidt, F. Int Z. Klin. Pham. Ther Tox. 1968, I, 461.

6. Kintz, P.; Ludes, B.: Mangin, P. J. Forensic Sci. 1992, 37, 72.

7. Balabanova, S.; Wei, B.; Rosing Gi; Scherer, Gi; Mayerhofer, C.: Chen, Z.; Zhang, W.; Rosenthal, J. Anthropol. Atz. 1996, 54, 341.

8. Deviprasad, G R.; D'Souza, F. Chem. Commun. 2000, 1915.

9. Craig Medical Distribution Inc.: Vista, CA, 92081, LSA.

10. Pal, T.: Narayanan, V. A.; Stokes, D. L.; Vo-Dinh, T. Anal. Chim. Acta 1998, 368, 21.

11. Ishikawa, H.; Imai, Y,; Kurokawa, Y, Vib. Spectrosc. 1995, \&, 445.

12. Barber, T. E.; List, M. S.; Haas, J. W.; Wachter, E. A. Appl. Spectrosc. 1994, 48, 1423.

13. Bell, S. E. .T. Sirimuthu, N. M. S. Anahys 2004, /29, 1032.

14. Laserna. T. T. Anol. Chim. Acto 1993, $283,607$.

15. Taylor, G. T.: Sharma, S. K.; Mohanan, K. Appl. Spectrosc. 1990. 44,635 .
16. Park, T.; Lee, S.; Seong, G H.; Lee, E. K.; Kim, Y. S.; Ji, W. H.; Hwang, S. Y.; Gweon, D. G.; Lee, S. Lab, Chip 2005, 5, 437.

17. Yea, K.; Lee, S.; Kyong, J. B.; Choo, J.; Lee, E. K.; Joo, S.-W.; Lee, S. Anatyst 2005, 130, 1009.

18. Lee, D.: Seong, G. H.; Choo, J.: Gweon, D. G.; Lee, \$. Appl. Spectrosc. in press.

19. Leopold, N.; Lendl, B. J. Phys. Chent. B 2003, 107, 5723.

20. Kin, D. J.; Oh, H. J.; Lee, S.; Park, T.; Choo, J. Analyst 2005, /30, 293.

21. Park, T.; Lee, M.; Choo, J.; Kim, Y. S.; Lee, E. K.; Kim, D. J.: Lee, S. Appl. Spectrosc. 2004, $58,1172$.

22. Heule, M.: Manz, A. Lab. Chip 2004, 4, 506.

23. Jeon, S.; Woo, J.; Kyong. J. B.; Choo, J. Butl. Kor. Chent. Soc. $2001,22,1264$.

24. Jeon, S.; Choo, J.; Kim, S.; Kwon, Y.; Kim, J.; Lee, Y.; Chung, H. J. Hol. Strict. 2002, 609, 159. 